\title{
IDENTIDAD POLÍTICA Y PARTICIPACIÓN: LA INICIATIVA CIUDADANA EUROPEA
}

\author{
Leyre Burguera Ameave
}

\begin{abstract}
SUMARIO: 1. INTRODUCCIÓN. 2. IDENTIDAD EUROPEA Y CIUDADANÍA «POST-NACIONAL»: DE LA INTEGRACIÓN A LA AUTOCOMPRENSIÓN. 3. INICIATIVA CIUDADANA EUROPEA: REGULACIÓN, IMPLEMENTACIÓN E IMPACTO. 4. Algunas ReFleXiones y CONSIDERACIONES CRÍTICAS. 5. A MODO DE CONCLUSIÓN. 6. REFERENCIAS BIBLIOGRÁFICAS.
\end{abstract}

\section{INTRODUCCIÓN}

Desde los inicios del proceso de integración europea ha habido un concepto que se ha manejado y discutido intensamente, que en el fondo está en el sustrato de la misma idea que tenían los padres fundadores de la Unión Europea (UE): el concepto de ciudadanía europea. Sin duda, aunque se ha avanzado notablemente en la construcción de la integración europea, no parece que se haya logrado el mismo nivel de éxito en lo que se refiere al sentimiento y al reconocimiento de la ciudadanía europea.

En los últimos años, venimos asistiendo a un proceso de conformación de la ciudadanía europea basado en un enfoque nacional meramente agregativo, que ha resultado ser ejemplo y síntoma de la propia evolución política de la UE. No obstante, con el rediseño institucional articulado gracias a la aprobación del Tratado de Lisboa, la UE ha configurado una nueva herramienta de democracia participativa transnacional, la iniciativa ciudadana europea, que podría permitir un cierto avance en la pretensión sería la de devolver al ciudadano su protagonismo como agente de cambio y de legitimación del proceso de construcción europeo. Desde este punto de vista, nos parece de sumo interés analizar la iniciativa ciudadana europea en el proceso legislativo como una oportunidad para lograr una europeización horizontal, capaz de integrar a todos los ciudadanos bajo una concepción inclusiva de identidad europea. 


\section{IDENTIDAD EUROPEA Y CIUDADANÍA «POST-NACIONAL»: DE LA INTEGRACIÓN A LA AUTOCOMPRENSIÓN}

Europa siempre ha sentido la necesidad de reforzar la idea de la consecución de una verdadera democracia transnacional como parte de su proceso de construcción, y para ello no ha dejado de trabajar articulando mecanismos que reequilibraran las fuerzas entre las distintas instituciones que la integran.

Íntimamente relacionada con esta preocupación, a ella se ha unido la necesidad de elaboración de una noción de ciudadanía europea que superase el vínculo de nacionalidad como principal criterio de adscripción. Esta limitación sustentada bajo principios territoriales constriñe un concepto que, a juicio de muchos, necesita crear un vínculo supranacional que simbolice un verdadero proceso de integración.

En realidad, estas dos inquietudes, que están en la base de las constantes llamadas de atención sobre el problema del déficit democrático de las instituciones europeas y su progresiva deslegitimación, sacan a relucir la dificultad señalada por Sánchez-Cuenca de cuál deba ser el modo de enfocarlo. Podemos hacerlo poniendo toda nuestra atención sobre la ausencia de un demos, un pueblo, en el que las distintas ciudadanías europeas se reconozcan, o bien entender que el problema se debe a un deficiente diseño institucional que impide una mayor participación ciudadana en la toma de decisiones ${ }^{1}$. Es evidente que, aunque complementarias, estas dos vías de análisis requieren de un esfuerzo teórico y práctico notablemente dispar. No obstante, en esta breve introducción quisiera destacar algunas consideraciones en torno a los dos enfoques propuestos: el enfoque horizontal (apelación a un demos europeo) y el enfoque vertical (apelación a un rediseño institucional que permita una mayor integración ciudadana en la conformación de la UE).

En primer lugar, enfocar los esfuerzos en la construcción de un demos orgánico europeo supone aludir a la noción misma de identidad y a la necesidad de una edificación ex novo que permita superar las narrativas históricas de cada Estado nacional. En este sentido, el diagnóstico actual de la situación en la UE resulta evidente, y es que hoy en día no existe un demos europeo. Sin embargo, a juicio de Habermas, este objetivo no es imposible de lograr si aceptamos la premisa inicial de que la conciencia nacional es una construcción artificial enteramente moderna y que la historia nacional se elaboró con la ayuda de juristas, historiadores, lingüistas, etc., y se produjo soterradamente gracias a los procesos educativos, a los medios de comunicación, etc ${ }^{2}$. Así pues, cabe plantearse si en las actuales circunstancias ese proceso de

${ }^{1}$ SÁnchez Cuenca, I., «El déficit democrático de la Unión Europea», en LlamazARES, I. y REINARES, F. (Eds.), Aspectos políticos y sociales de la integración europea, Tirant lo Blanch, Valencia, 1999, pp. 93-94.

${ }^{2}$ Habermas, J., El occidente escindido, Trotta, Madrid, 2006, pp. 79-80. 
aprendizaje de una identidad europea es posible, y para este autor lo es, siempre y cuando se cumplan unos supuestos empíricos: «a) es necesario que exista una sociedad de ciudadanos europeos; b) hay que construir un espacio público político que abarque a toda Europa, y c) hay que crear una cultura política que pueda ser compartida por todos los ciudadanos de la Unión Europea $»^{3}$.

Evidentemente esto conlleva un proceso de aprendizaje lento y complejo que debe superar la denominada por Habermas como integración funcional, que ha sido la fórmula empleada hasta ahora para la conformación de la UE, y que ha consistido en una suerte de inercia intergubernamental a favor de una Unión política en la que no se han anticipado debidamente ni consecuencias ni resultados, y donde tampoco se han asumido responsabilidades. En contraposición, este autor aboga por favorecer «una integración normativa de los ciudadanos que hiciese posible que los objetivos se fijasen en común por encima de las fronteras nacionales» ${ }^{4}$. Para lograrlo, sería necesario un nuevo escenario donde se pueda dar la confluencia de distintas anticipaciones (una Carta Europea que anticipe las competencias que tendría una futura Constitución europea, unos partidos políticos que debatieran sobre asuntos europeos sentando las bases para conformar verdaderos partidos europeos, una opinión pública europea que de voz a una sociedad civil europea, etc.) que en un proceso circular de apoyo y estímulo mutuo, sentase las bases para la consecución de una conciencia europea común capaz de crear una democracia postnacional ${ }^{5}$.

Con la mirada puesta en este objetivo, gracias a la sentencia sobre el Tratado de Maastrich del Tribunal constitucional alemán ${ }^{6}$ se dio un incipiente paso en la discusión sobre la identidad europea asociada a la idea de la democracia, ya que como señala Closa, se hacía mención y debatía la cuestión de la democracia, y los rasgos preexistentes a ella, destacando la identidad como elemento constitutivo de la nación sobre la cual se superpone la democracia ${ }^{7}$. En esta línea de discusión y de enfoque del problema, se han mantenido autores como Smith, que entiende que «(...) la identidad (nacional o cultural) emerge como elemento fundacional de la democracia y la ausencia de este elemento predemocrático, la identidad europea, aparece como un obstáculo para una UE democrática» ${ }^{8}$. De ahí que haya sido una tarea constante, por

${ }^{3}$ Habermas, J., Tiempo de transiciones, Trotta, Madrid, 2004, p. 126.

${ }^{4}$ Habermas, J., El occidente...op.cit., pp. 71-72.

5 Habermas, J., La constelación posnacional, Paidós, Barcelona, 2000, pp. 133-134.

${ }^{6}$ Ver casos 2 BvR 2134/92 y 2159/92 Manfred Brunner y otros.

7 Closa, C., «La ciudadanía europea», en Llamazares, I. y Reinares, F. (Eds.), Aspectos políticos y sociales de la integración europea, Tirant lo Blanch, Valencia, 1999, p. 87.

8 SMith, A., «National Identity and the Idea of European Unity», International Affairs, vol.68, n 1, 1992, pp. 55-76. 
parte de la doctrina, la búsqueda de esa identidad basada en «el tradicionalismo (o la fundación de la unidad europea en un hecho espiritual, por ejemplo, la religión), el modernismo y el constructivismo (la creación de un espacio cultural homogéneo a través de las tecnologías de la comunicación)»».

Pese a estos esfuerzos, lo cierto es que desde hace años hemos insistido en ese enfoque nacional meramente agregativo de la ciudadanía, por eso no resulta extraño observar cómo el proceso de conformación de la ciudadanía europea ha resultado ser ejemplo y síntoma de la propia evolución política de la UE. Como destaca Beck, a día de hoy seguimos concibiendo la identidad europea como un modelo colectivo, a la vez que agregativo y repartido, que tiene su sustrato en la identidad nacional de los distintos países que conforman la $\mathrm{UE}^{10} \mathrm{y}$, con base en esta percepción, es normal que sigamos recurriendo al campo semántico del movimiento para hacer mención al proceso de «construcción de Europa»"

En atención al uso de este campo semántico que apela a la construcción de la UE, es importante destacar la oportunidad que nos brinda el análisis del enfoque vertical del problema para conformar una identidad europea, y que consiste en observar las posibles consecuencias que se pueden producir al implementar la iniciativa ciudadana europea dentro de su proceso de rediseño institucional.

En relación a este complejo objetivo, considero que quizá una vía indirecta pero más sencilla de lograr una europeización horizontal, capaz de permitir recobrar fuerza a una sociedad civil que se conciba asimisma como ciudadanía europea, puede hallarse en el rediseño institucional que articule cauces efectivos y eficaces de participación ciudadana.

De ahí que estime que para lograr un concepto de ciudadanía europea verdaderamente inclusivo, que supere la idea de ciudadanía «post-nacional», hemos de ser capaces de articular efectivos instrumentos de participación política basados en dinámicas transnacionales que queden supeditados a unas condiciones estructurales de participación que trasciendan el ámbito nacional. Debemos huir, por tanto, de la implícita influencia de la «lógica de los Estados», que delimita y condiciona de manera determinante cualquier intento de participación activa ciudadana. En esta tarea hemos de ser capaces de construir una dinámica propia que evite lo que en su día ya señaló Fraile Ortiz, y es que siga prevaleciendo el diseño instrumental del concepto de ciudadanía al servicio de la legitimación de todo el proceso de construcción

${ }^{9}$ Closa, C., op. cit., pp. 88-89.

${ }^{10}$ Beck, U. y Grande, E. (2006): La Europa Cosmopolita. Sociedad y política en la segunda modernidad, Paidós, Barcelona, 2006, pp. 148-150.

${ }_{11}$ Resulta interesante observar la utilización de metáforas y palabras recurrentes como: «ponerse en camino», «abrir «calles», «caminos», «rutas» o vías aún no del todo «transitables», etc. Ibídem, p. 152. 
de Europa, de manera que finalmente quede desprovisto de apoyo social efectivo $^{12}$. Para lograr este propósito, la iniciativa ciudadana europea se postula como un esfuerzo esperanzador que merece ser analizado.

\section{INICIATIVA CIUDADANA EUROPEA: REGULACIÓN, IMPLEMENTACIÓN E IMPACTO}

La aprobación del Tratado de Lisboa ha dispuesto que todo ciudadano tenga derecho a participar en la vida democrática de la Unión mediante la figura de la iniciativa ciudadana europea. Un importante paso en la construcción horizontal europea que denota la importancia del poder legislativo como vector de unión.

Respecto al marco legislativo en el que se inscribe la iniciativa ciudadana europea, cabe destacar, en primer lugar, que nos encontramos ante un derecho contemplado en el artículo 11.4 del Tratado de la Unión Europea cuyo ejercicio, procedimientos y requisitos ha sido desarrollado a través del Reglamento (UE) n ${ }^{\circ}$ 211/2011 del Parlamento Europeo y del Consejo, de 16 de febrero de 2011 y del Reglamento de Ejecución (UE) n ${ }^{\circ} 1179 / 2011$ de la Comisión, de 17 de noviembre de 2011, por el que se establecen especificaciones técnicas para sistemas de recogida de apoyos a través de páginas web.

Se trata de un proceso de conformación de la iniciativa legislativa ciudadana que se articula en torno a unos sencillos pasos que, a continuación, brevemente detallaré:

1. Preparación y establecimiento del comité de ciudadanos:

- Las iniciativas deben plantearse a propuesta de un comité de ciudadanos, integrado por siete o más ciudadanos de la UE que tengan edad suficiente para votar en las elecciones al Parlamento Europeo (18 años excepto en Austria, donde se vota a partir de los 16) y residan en al menos siete Estados miembros distintos. Aunque no se exige que sean ciudadanos de siete Estados miembros diferentes, sí deben tener la nacionalidad de algún Estado miembro de la UE.

- Este comité se considera como «organizador» oficial de la iniciativa y es el responsable de gestionarla a lo largo de todo el procedimiento.

- El comité debe designar de entre sus miembros a un representante y un sustituto que hablen y actúen en nombre del comité. Serán las personas de contacto que servirán de puente entre el comité de ciudadanos y la Comisión a lo largo de todo el procedimiento. Los di-

12 Fraile Ortíz, M., El significado de la ciudadanía europea, CEPC, Madrid, 2003, p.22. También resulta interesante consultar: FraILE ORTíz, M., «La ciudadanía europea (entre paréntesis)», Revista Fundamentos, Volumen 7: El Pueblo del Estado. Nacionalidad y Ciudadanía en el Estado Constitucional-Democrático, 2012, pp. 311-357. 
putados al Parlamento Europeo no pueden contabilizarse para alcanzar el mínimo de siete ciudadanos requerido para constituir un comité ciudadano.

2. Registro de la iniciativa propuesta (plazo máximo 2 meses). Para poder inscribir su iniciativa en el registro, los organizadores deben facilitar la siguiente información en una de las lenguas oficiales de la UE:

- el título de la iniciativa ciudadana propuesta (máximo 100 caracteres);

- su objeto (máximo 200 caracteres);

- los objetivos de la iniciativa sobre la que la Comisión debe decidir (máximo 500 caracteres);

- las disposiciones de los Tratados consideradas pertinentes por los organizadores de la acción propuesta;

- el nombre completo, la dirección postal, la nacionalidad y la fecha de nacimiento de los siete miembros del comité de ciudadanos, indicando específicamente el representante y el sustituto, así como sus direcciones de correo electrónico y números de teléfono;

- documentos que demuestren el nombre completo, la dirección postal, la nacionalidad y la fecha de nacimiento de cada uno de los siete miembros del comité de ciudadanos;

- todas las fuentes de financiación y apoyo a la iniciativa ciudadana propuesta (conocidas en el momento del registro) superiores a 500 euros por año y patrocinador.

3. Certificación del sistema de recogida a través de páginas web (plazo máximo 1 mes). Los organizadores que deseen recoger declaraciones electrónicas de apoyo deben crear un sistema de recogida a través de páginas web, accesible a través de su sitio web, que se ajuste a los requisitos técnicos y de seguridad contemplados en el artículo 6.4 del Reglamento sobre la iniciativa ciudadana, así como a las especificaciones técnicas detalladas que se establecen en un Reglamento específico (Reglamento de Ejecución (UE) n. ${ }^{\circ}$ 1179/2011 de la Comisión). El objeto de dichos requisitos es garantizar, en particular, la seguridad de la recogida de los datos y de su almacenamiento en el sistema.

Cuando hayan creado un sistema de recogida a través de páginas web que cumpla plenamente los requisitos señalados más arriba, los organizadores deberán solicitar su certificación por la autoridad nacional competente del Estado miembro en el que se vayan a almacenar los datos. Para ello, tendrán que facilitar a la autoridad competente la documentación necesaria.

4. Recogida de declaraciones de apoyo electrónicas o en papel (plazo máximo 12 meses). Una vez confirmada la inscripción de la iniciativa propuesta, los organizadores pueden empezar a recoger las declaracio- 
nes de apoyo de los ciudadanos. Dispondrán de 12 meses para obtener el millón de declaraciones exigidas, alcanzando los mínimos establecidos en al menos siete Estados miembros.

5. Verificación de las declaraciones de apoyo (plazo máximo 3 meses). Tras recoger las declaraciones de apoyo necesarias, los organizadores deben pedir a las autoridades nacionales competentes de los Estados miembros donde se haya llevado a cabo la recogida que certifiquen el número de declaraciones válidas obtenidas en cada Estado.

6. Presentación de la iniciativa ante la Comisión. Tras haber obtenido de las autoridades nacionales competentes los certificados (siete como mínimo) que acreditan la recogida del número necesario de declaraciones de apoyo (un millón en total, alcanzando los mínimos establecidos en al menos siete Estados miembros), los organizadores pueden presentar su iniciativa ante la Comisión, junto con la información relativa a los apoyos y la financiación que hayan recibido.

7. Examen, audiencia pública en el Parlamento Europeo y respuesta de la Comisión (plazo máximo 3 meses). En el plazo de tres meses a partir de la presentación de la iniciativa los organizadores se reunirán con representantes de la Comisión para explicar detalladamente las cuestiones que plantea su iniciativa y lo podrán hacer en una audiencia pública organizada en el Parlamento Europeo. La Comisión aprobará un documento oficial que especifique, si las hay, las medidas que tenga la intención de proponer en respuesta a la iniciativa ciudadana y los motivos por los que haya decidido actuar o no hacerlo.

Por último, si finalmente la Comisión decide seguir adelante con la iniciativa, se pondría en marcha el procedimiento legislativo.

Respecto a su implementación e impacto, merece la pena destacarse la variedad de propuestas presentadas hasta el momento, ya que toman en consideración aspectos tan dispares como el derecho al agua, la neutralización de las sociedades pantalla, la necesidad de establecer un plan europeo extraordinario que promueva el desarrollo sostenible y el empleo, el incremento del pluralismo de los medios de comunicación, la suspensión del paquete de energía y cambio climático de la UE, etc.

Otra cuestión es su desigual suerte dentro del procedimiento de tramitación diseñado por el Reglamento (UE) n. ${ }^{\circ}$ 211/2011 del Parlamento Europeo $\mathrm{y}$ del Consejo. A este respecto, podemos establecer varios itinerarios ${ }^{13}$ :

En primer lugar están las iniciativas que se denominan obsoletas, y que son aquellas que bien porque no han obtenido el número necesario de declaraciones de apoyo en el plazo de un año (es el caso de las iniciativas: «Aca-

${ }^{13}$ En este sentido acudir a $<$ http://ec.europa.eu/citizens-initiative/public/initiatives $>$ (última consulta 14/11/2014). 
bemos con el Ecocidio en Europa: Una Iniciativa Ciudadana para dar Derechos a la Tierra», «Renta Básica Incondicional (RBI) - Explorando un camino hacia las condiciones de bienestar emancipatorias en la UE», «Single Communication Tariff Act», etc.) o bien porque han sido retiradas por sus organizadores (es el caso de las iniciativas: «MOVEUROPE», «Turn me Off!», «Teach for Youth - Upgrade to Erasmus 2.0», «Kündigung Personenfreizügigkeit Schweiz», «Iniciativa Europea a favor del Pluralismo en los Medios de Comunicación», «End Ecocide in Europe: A Citizens' Initiative to give the Earth Rights», «Let me vote», «Directiva Europea sobre el Bienestar de las Vacas Lecheras», etc.) se encuentran fuera del procedimiento.

En segundo lugar están aquellas iniciativas cuya recogida de firmas está cerrada y que por tanto:

a) Se hallan a la espera de ser presentadas ante la Comisión (es el caso de la iniciativa: «30 km/h - por unas calles habitables!» o «Stop Vivisection»).

b) Ya han sido presentadas ante la Comisión y esperan ser atendidas por ella.

c) Ya han sido atendidas por la Comisión y además se han hecho públicas las conclusiones. En este supuesto se encuentran dos iniciativas, una que finalmente ha sido rechazada («Uno de nosotros») y otra que no («El derecho al agua y el saneamiento como derecho humano $¡ E l$ agua no es un bien comercial, sino un bien público!»), por lo que puede considerarse la primera iniciativa ciudadana europea que ha superado todos los trámites con éxito.

Por último, están las iniciativas abiertas debido a que permanecen a la espera de que concluya el plazo de recogida de apoyos, una vez superados los trámites previos de registro e inscripción. Es el caso, por ejemplo, de la iniciativa: «Por una Europa más justa, neutralicemos las sociedades pantalla» cuyo plazo de recogida de firmas concluirá el próximo 1/10/2015.

En definitiva, podemos afirmar que pese al corto espacio de tiempo transcurrido desde su implementación, su acogida por parte de la ciudadanía ha sido muy favorable y receptiva. Una muestra más de que los ciudadanos exigimos poder participar más y mejor en la comunidad política supranacional de la que formamos parte.

\section{ALGUNAS REFLEXIONES Y CONSIDERACIONES CRÍTICAS}

Una vez que se ha realizado un breve panorama descriptivo de la iniciativa ciudadana europea, quisiera realizar una sucinta valoración de este instrumento desde distintas perspectivas de análisis.

Desde un plano más amplio y genérico, es indudable que la puesta en marcha de este instrumento tiene una consideración positiva en cuanto que 
ha supuesto un notable avance en el reforzamiento de la idea de ciudadanía europea.

No en vano, en el horizonte político europeo siempre se ha tenido muy presente, tal y como señala Offe, que no era posible «un fortalecimiento de la capacidad de gobierno de las instituciones europea sin la ampliación formal de los fundamentos de su legitimidad democrática» ${ }^{14}$.

En este empeño han fijado sus esfuerzos las instituciones europeas, de forma que han visto en la propuesta de la iniciativa ciudadana europea, un modo de ampliar la capacidad de acción política de los ciudadanos. Esto permite acercarnos al concepto jurídico de autolegislación propugnado por Habermas que considera que «debe adquirir una dimensión política hasta transformarse en el concepto de una sociedad democrática que actúa sobre sí misma» ${ }^{15}$.

Los ciudadanos europeos en la medida en que se consideren miembros y participantes de un proyecto común, podrán percibir que su identidad trasciende del ámbito nacional y se integra en una nueva identidad que no queda superpuesta ni supeditada a la anterior. Al contrario, nuestra identidad europea no puede ser sin más algo que construyamos como un mero agregado de identidades nacionalidades sino que debe ser, a la vez, nuestro propio proyecto de futuro. $\mathrm{Y}$ en este sentido, considero la iniciativa ciudadana europea como un importante paso a tener en cuenta.

Desde un enfoque más próximo a su concreta articulación normativa, la valoración genérica positiva requiere de unos matices críticos.

Cuando hacemos referencia a la iniciativa ciudadana europea, en realidad estamos aludiendo a la posibilidad de que un grupo de ciudadanos formule una propuesta a la Comisión Europea para que en el ámbito de sus competencias plantee un texto legislativo al Parlamento y al Consejo.

De esta primera aproximación a su concepción podemos extraer dos consideraciones iniciales que van a supeditar de manera determinante su articulación. La primera es que, tal y como estipula el artículo 2 del Reglamento (UE) n. ${ }^{\circ}$ 211/2011 del Parlamento Europeo y del Consejo, de 16 de febrero de 2011, no existe una obligación formal de presentar la propuesta tal y como fue formulada, por tanto, la Comisión no queda vinculada al contenido de la iniciativa como así parece evidenciarlo la ambigua expresión de: «(...)propuesta adecuada sobre las cuestiones sobre las que los ciudadanos estimen que se requiere un acto legislativo de la Unión para los fines de la aplicación de los Tratados» que contiene el Reglamento. Y en directa relación con esta cuestión, es por tanto evidente, que las limitaciones materiales son muchas, ya que solo cabe plantear una iniciativa en el ámbito de las atribuciones de la Comisión. Un asunto que además plantea ciertos reparos si, como recuerda Elvira, tenemos en cuenta que la mayoría de las materias tienen un carácter

\footnotetext{
${ }^{14}$ Offe, C., Demokratie und Wohlfartsstaat, 1998, p. 27.

15 Habermas, J., La constelación ...op.cit., p. 83.
} 
compartido y que por tanto no son de exclusiva competencia comunitaria ${ }^{16}$. En este sentido, ¿tendrían algo que decir los parlamentos nacionales como órganos co-decisores?

De todo ello se deduce que nos encontramos ante un instrumento que ha quedado articulado a través de la necesaria intermediación de la Comisión. Por tanto, es notorio que pese a los constantes esfuerzos por delimitar el monopolio de la iniciativa legislativa por parte de la Comisión, este instituto de participación ha quedado en sus manos. Esta situación solo se nos antoja comprensible si no cuestionamos las específicas competencias que tiene la Comisión en orden a la propuesta legislativa ${ }^{17}$, pero desde una perspectiva crítica, quizá no sea una osadía proyectar una necesidad de cambio en orden a la consecución de una UE cuya gobernanza se aproxime al sistema parlamentario tradicional.

En este escenario político, el reequilibrio institucional debe decantarse por una recuperación de cierto protagonismo parlamentario donde el déficit democrático sea en parte solventado por institutos como la iniciativa ciudadana europea, que permitan una mayor presencia ciudadana en el devenir y construcción de Europa.

De momento, tal y como queda configurada la iniciativa, las cuestiones procedimentales parecen evidenciar otro importante problema, y es que suponiendo que la iniciativa ciudadana europea pudiera residenciarse en sede parlamentaria, a día de hoy su regulación parece incidir en los aspectos esencialistas de la nacionalidad, una circunstancia que podría socavar el carácter transnacional del Parlamento Europeo.

Ejemplos de esta percepción los encontramos en el propio Reglamento mencionado cuando regula la condición diferenciada de «firmante» y «organizador» y establece para ello unos requisitos ligados a la nacionalidad (art. 3) y no a la residencia, que trata de matizar al exigir que, para garantizar la representación del «interés de la unión», el comité esté integrado por siete o más ciudadanos que provengan de diferentes Estados de la UE. En este sentido, se han presentado objeciones sobre cómo articular ese «interés general» ya que la exigencia de que sean los ciudadanos de tan sólo siete Estados miembros los necesarios para su conformación suscitó cierto debate ${ }^{18}$ antes de su concreción reglamentaria (entre otras cuestiones se discutió sobre la ausencia de criterios geográficos en su determinación), a la vez que no ha evitado las críticas sobre la posibilidad de que este instrumento quede en manos de colectivos muy bien organizados (o incluso grupos de presión) con respaldo económico suficiente para llevarlo a cabo.

${ }^{16}$ Bilbao, J. M., y VinTró, J., Participación ciudadana y procedimiento legislativo: de la experiencia española a la iniciativa ciudadana europea, Centro de Estudios Políticos y Constitucionales, Madrid, 2011, p. 104.

${ }_{17}$ Gómez SÁnchez, Y., «La iniciativa ciudadana en la Unión Europea», Panorama Social, núm.17, 2013, p. 65.

${ }^{18}$ Bilbao, J. M., y VinTRó, J., op. cit., pp. 64-65. 
También otro aspecto procedimental asociado a la nacionalidad se evidencia cuando se determina que sea una autoridad nacional la que certifique el sistema de recogida de firmas (art. 6) y verifique las declaraciones de apoyo (art. 8) que, en todo caso, requieren de un número mínimo en función del Estado (art.7 y anexo I) y que si son recabadas por la vía electrónica, se someterán a la normativa de protección de datos de cada país (art. 12) ${ }^{19}$. En este sentido, hubiera sido deseable que se creara para el proceso de verificación una «firma electrónica europea». Una oportunidad que habría reforzado la articulación y consecución de una efectiva ciudadanía europea.

Además, debemos destacar que, el tipo de datos personales que se nos van a exigir a la hora de rellenar los formularios de declaración de apoyo a una propuesta van a depender de la legislación nacional de cada Estado (anexo III). Una cuestión conflictiva que, sin duda, evidencia un poco más, la esencia nacional en la configuración procedimental de este instrumento de participación ciudadana.

Otras posibles valoraciones críticas a la iniciativa ciudadana europea consistirían en la falta de exigencia de un texto articulado junto con la mera propuesta, (lo que deja a este instrumento de participación la calificación jurídica de mero derecho de petición y no verdadera iniciativa legislativa $\left.{ }^{20}\right)$, la ausencia de previsión en el Reglamento de la oportunidad de su defensa (e incluso la introducción de enmiendas) por parte del Comité organizador en el trámite parlamentario posterior o la falta de previsión legal sobre la posibilidad de volver a presentar una iniciativa fallida.

Todas estas consideraciones sujetas a debate, no restan importancia a su apreciación como importante instrumento de participación política transnacional, de modo que no debemos de olvidar que nos encontramos ante lo que Bilbao destaca como la primera herramienta de participación directa adoptada por una organización supranacional ${ }^{21}$, y eso merece un reconocimiento más allá de su análisis prospectivo.

\section{A MODO DE CONCLUSIÓN}

Pese a las reticencias iniciales que siempre existen en torno a la articulación de fórmulas activas de participación ciudadana, Europa ha hecho un importante esfuerzo, a través de la iniciativa ciudadana europea, por consoli-

${ }^{19}$ Esta cuestión puede plantear cuestiones controvertidas en el futuro. En este sentido ver Cotino Hueso, L., «El Reglamento de la Iniciativa Ciudadana Europea de 2011. Su especial regulación de la recogida de apoyos vía internet y de la protección de datos de los ciudadanos», Revista de derecho político, n. ${ }^{\circ}$ 81, 2011, pp. 323-378.

${ }^{20}$ BilbaO, J. M., y Vintró, J., op. cit., p. 112.

${ }^{21}$ Bilbao, J. M., y Vintró, J., Ibídem, p. 98. 
dar la idea de ciudadanía europea como sujeto político con entidad propia no asociada estrictamente a la nacionalidad.

Este objetivo ha pretendido socavar los aspectos esencialistas de la nacionalidad en la construcción de la identidad europea pero, lejos de lograrlo, ha evidenciado la dificultad que entraña la conformación del estatuto de ciudadano europeo.

Pese a todo, la iniciativa ciudadana europea, puede considerarse un importante instrumento para la democratización de Europa que trate de salvar la distancia existente entre las instituciones y los ciudadanos europeos. De este modo, su implementación puede mejorar la integración de Europa «desde abajo», desplegando todo su potencial creador de comunidad.

Por ello estimo que nos encontramos ante un significativo paso en la construcción de la ciudadanía europea que parece apostar por esta vía «indirecta» que consiste, básicamente, en el rediseño institucional que introduce la iniciativa ciudadana en el proceso legislativo.

\section{REFERENCIAS BIBLIOGRÁFICAS}

Beck, U. y Grande, E., La Europa Cosmopolita. Sociedad y política en la segunda modernidad, Paidós, Barcelona, 2006.

Bilbao, J. M., VInTRó, J., Participación ciudadana y procedimiento legislativo: de la experiencia española a la iniciativa ciudadana europea, Centro de Estudios Políticos y Constitucionales, Madrid, 2011.

Closa, C., «La ciudadanía europea», en Llamazares, I. y Reinares, F. (Eds.), Aspectos politicos y sociales de la integración europea, Tirant lo Blanch, Valencia, 1999.

Cotino Hueso, L., «El Reglamento de la Iniciativa Ciudadana Europea de 2011. Su especial regulación de la recogida de apoyos vía internet y de la protección de datos de los ciudadanos», Revista de derecho político, n. ${ }^{\circ} 81,2011$, pp. 323-378.

Fraile Ortíz, M., El significado de la ciudadanía europea, CEPC, Madrid, 2003.

GARCÍA GuitiÁN, E., «Problemas de la representación política», en ARTETA, A., GARCÍA Guitián, E. y MAIz, R. (eds.), Teoría política: poder, moral, democracia, Alianza editorial, Madrid, 2003.

Gómez SÁnchez, Y., «La iniciativa ciudadana en la Unión Europea», Panorama Social, núm.17, 2013.

HABERMAS, J., La constelación posnacional, Paidós, Barcelona, 2000.

- Tiempo de transiciones, Trotta, Madrid, 2004.

El occidente escindido, Trotta, Madrid, 2006.

OFFe, C., Demokratie und Wohlfartsstaat, 1998.

Peña, J., «La ciudadanía», en Arteta, A., García Guitián, E. y Maiz, R. (eds.), Teoría política: poder, moral, democracia, Alianza editorial, Madrid, 2003.

SÁnCHEZ CUENCA, I., «El déficit democrático de la Unión Europea», en LlAMAZARES, I. y ReInARES, F. (Eds.), Aspectos políticos y sociales de la integración europea, Tirant lo Blanch, Valencia, 1999.

SмIтн, A., «National Identity and the Idea of European Unity», en International Affairs, vol.68, n. ${ }^{\circ} 1,1992$. 
TitTLE: Political identity and participation: the european citizens' initiative.

RESUMEN: La Unión Europea es consciente de la necesidad de articular fórmulas activas de participación que devuelvan a los ciudadanos su protagonismo como agentes de cambio y de legitimación del proceso de construcción europeo. El rediseño institucional articulado gracias a la aprobación del Tratado de Lisboa, ha configurado una nueva herramienta de democracia participativa transnacional, la iniciativa ciudadana europea, que podría permitir un cierto avance en la europeización horizontal, capaz de integrar a todos los ciudadanos bajo una concepción inclusiva de identidad europea. Desde esta perspectiva, se trata de analizar su regulación e implementación como potencial creador de comunidad política.

PALABRAS CLAVE: Europa, ciudadanía, democracia participativa e iniciativa legislativa.

ABSTRACT: The European Union is aware of the need to create active way of participation that returned to the citizens for their role as agents of change and legitimization of the European construction process. The institutional redesign articulated thanks to the adoption of the Lisbon Treaty, has set a new tool of transnational participatory democracy, the European Citizens' Initiative (ECI), which could allow some movement in the horizontal Europeanization, able to integrate all citizens under an inclusive conception of European identity. From this perspective, it is analyze his regulation and implementation as creative potential of political community.

KEYWORDS: Europe, citizen, participatory democracy and legislative initiative.

RECIBIDO: 11.11 .2014

ACEPTADO: 26.11.2014 